\title{
LEVANTAMENTO E IDENTIFICAÇÃO DE PARASITÓIDES DE DÍPTEROS MINADORES EM PLANTAS DE CRESCIMENTO ESPONTÂNEO EM POMAR ORGÂNICO DE CITROS EM MONTENEGRO, RS, BRASIL
}

\author{
J.P. dos Santos ${ }^{1}$, F.K. Dal Soglio ${ }^{2}$, L.R. Redaelli², E. Foelkel ${ }^{2}$
}

${ }^{1}$ EPAGRI, Estação Experimental de Caçador, Laboratório de Entomologia, CP591,CEP89500-000, Caçador, SC, Brasil. E-mail: janapereira@epagri.sc.gov.br

\section{RESUMO}

\begin{abstract}
Este trabalho teve como objetivos realizar o levantamento e a identificação dos parasitóides associados aos dípteros minadores presentes na vegetação de crescimento espontâneo, em pomar orgânico de citros, e verificar se estes parasitóides são os mesmos relatados para o minador-dasfolhas-dos-citros (Phyllocnistis citrella). O trabalho foi conduzido em Montenegro, RS, em um pomar de tangoreiro Murcott. Realizaram-se amostragens quinzenais, de maio de 2003 a maio de 2004, coletando-se em cada ocasião 60 unidades de amostra. Cada unidade era constituída de todas as folhas com minas contidas na área delimitada por um aro de $0,28 \mathrm{~m}^{2}$, que era jogado na linha e na entrelinha de 30 árvores sorteadas. Nolaboratório as folhas foram mantidas, individualmente, em placas de Petri até a emergência dos parasitóides ou das moscas. Foram registradas 15 espécies demicroimenópteros: setedeEulophidae(Closterocerus coffeellae Ihering, Closterocerus sp., Chrysocharis tristis Hansson, Chrysocharis vonones Walker, Chrysocharis sp. 1, Chrysocharis sp. 2 eNeochrysocharis sp.), sete de Braconidae (Opius sp. 1, Opius sp. 2, Opius sp. 3, Opius sp. 4, Opius sp. 5, Centistideasp. 1, Centistideasp. 2) e uma de Figitidae, (Agrostocynips clavatus, Díaz). De acordo com os resultados, verificou-se que algumas espécies registradas no estudo pertencentes aos gêneros Closterocerus, Chrysocharis e Neochrysocharis são relatadas como parasitóides de P. citrella.
\end{abstract}

PALAVRAS-CHAVE: Inimigos naturais, Diptera, produção orgânica, Agromyzidae.

\section{ABSTRACT}

IDENTIFICATION OF PARASITOIDS OF DIPTEROUS LEAFMINERS PRESENT IN SPONTANEOUS VEGETATION IN AN ORGANIC CITRUS ORCHARD IN MONTENEGRO, RS, BRAZIL. The present study was aimed to survey and identify the parasitoids associated to dipterous leafminers occurring on spontaneous growing plants in an organic citrus orchard and to verify if these parasitoid species are the same ones reported for the citrus leafminer (Phyllocnistis citrella).The work was carried out in Montenegro, state of Rio Grande do Sul, Brazil, in a Murcott orchard. Fortnightly samplings, from May/2003 to May/2004, were carried out. On each occasion, 60 sampling units were collected. Each sampling unit consisted of all leaves with mines contained in an area delimited by a $0.28 \mathrm{~m}^{2}$ frame, thrown in and between lines aside each one of 30 randomly selected trees. In thelaboratory, the leaves were kept individually in Petri dishes until the emergence of parasitoids or flies. Fifteen species of hymenopterous were registered encompassing: Eulophidae (Closterocerus coffeellae Ihering, Closterocerussp., Chrysocharissp.1, Chrysocharis sp. 2, Chrysocharistristis Hansson, Chrysocharis vonones Walker and Neochrysocharis sp.); Braconidae (Opius sp. 1, Opius sp. 2, Opius sp. 3, Opius sp. 4, Opius sp. 5, Centistidea sp. 1 and Centistideasp. 2) and Figitidae (Agrostocynips clavatus, Díaz). According to the results, some species registered in the study, of the genera Closterocerus, Chrysocharis and Neochrysocharis, are reported parasitizing P. citrella.

KEY WORDS: Natural enemies, Diptera, organic production, Agromyzidae.

\section{INTRODUÇÃO}

As plantas de crescimento espontâneo nos agroecossistemas têmimportante papel nocontroleda erosão, na conservação da umidade e acúmulo de matéria orgânica no solo, na preservação da fauna, da flora, além da preservação de espécies de insetos benéficos (GLIESMANN et al., 1981). No controle biológico, a

\footnotetext{
${ }^{2}$ Universidade Federal do Rio Grande do Sul, Departamento de Fitossanidade, Porto Alegre, RS, Brasil.
} 
importância das plantas espontâneas num agroecossistema já havia sido apontada por VAN EMDEN (1964) como fonte alternativa de presas ou de hospedeiros, pólen, néctar e refúgios para inimigos naturais.

O minador-das-folhas-dos-citros, Phyllocnistis citrella (Stainton, 1856) (Lepidoptera: Gracillariidae), é uma importante praga da citricultura no Brasil. Os himenópteros parasitóides são os principais agentes de controle biológico de P. citrella (PENTEADO-Dias etal., 1997). Segundo Gravena (1996) e Verdú (1996), estes parasitóides podem ter como hospedeiros outros minadores que atacam plantas de crescimento espontâneo presentes em cultivos próximos aos citros.

Diptera é a segunda ordem em número de espécies com hábito minador, com destaque para a família Agromyzidae com representantes nos gêneros Liriomyza Mik e Agromyza Fallén (HespenheIde, 1991; Byers, 2002; Carletti, 2004). Várias espécies de agromizídeos estão associadas a plantas cultivadas (GALlo et al., 2002), sendo que muitas podem ser pragas secundárias em outras culturas e atacar plantas espontâneas (BYERs, 2002). Entretanto, no Brasil, poucos estudos têm sido desenvolvidos, especialmente com dípteros que se desenvolvem em plantas de crescimento espontâneo.

Parasitóides associados a moscas minadoras em diferentes regiões têm sido relatados; nos Estados Unidos da América, Schuster et al. (1991) encontraram os Braconidae Opius dissitus Muesebeck, $O$. bruneipes Gahan eO. mandibularis Gahan parasitando Liriomyza spp. PATEL et al. (2003) verificaram o Eulophidae Diglyphus intermedius (Girault) sobre $L$. trifolli (Burgess); em Cuba, LEÓN et al. (2000) registraram Diglyphus sp. associado a Liriomyza sp.; na Argentina, VAlladares; SAlvo (2001) constataram 29 espécies de Agromyzidae e 46 espécies de himenópteros parasitóides; no Brasil, PEREIRA et al. (2002) observaram os Braconidae Opius sp. parasitando L. huidobrensis (Blanchard); no Canadá, VenETTE et al. (2003) relataram Diglyphus spp. sobre várias espécies de Liriomyza e, na Colômbia, Cure; CANTOR (2003) observaram D. begini (Ashmead) associado a L. huidobrensis.

O conhecimento tanto sobre dípteros minadores quanto de seus parasitóides é ainda escasso e voltado especialmente para espécies que se desenvolvem em plantas de importância agrícola. Tendo em vista que a manutenção da vegetação espontânea, especialmente em pomares, é uma prática que vem sendo amplamente adotada, cabe a investigação sobre o seu potencial como reservatório de inimigos naturais. De acordo com PARRA et al. (2002) a utilização de práticas que favoreçam a ação de agentes de mortalidade natural de pragas nos agroecossistemas é de extrema importância, principalmente pela manipulação do ambiente, de modo a preservar e/ou aumentar as populações de inimigos naturais. Neste sentido, o presente estudo teve como objetivos realizar o levantamento ea identificação dos parasitóides associados aos dípteros minadores presentes na vegetação de crescimento espontâneo em um pomar orgânico de citros e verificar se estes parasitóides são os mesmos relatados para o minador-das-folhas-dos-citros (Phyllocnistis citrella), a fim de estabelecer estratégias de controle deste minador.

\section{MATERIAL E MÉTODOS}

O trabalho foi desenvolvido no Município de Montenegro $\left(29^{\circ} 68^{\prime} \mathrm{S}\right.$ e $\left.51^{\circ} 46^{\prime} \mathrm{O}\right)$, localizado no Vale do Rio Caí, RS. O estudo foi desenvolvido em um pomar do híbrido tangor Murcott (Citrus sinensis L. Osbeck x Citrus reticulata Blanco), com área de 0,6 ha, espaçamento entre plantas de 3,5 m e entrelinhas de $5 \mathrm{~m}$, com cerca de 370 plantas e 12 anos de idade.

Quinzenalmente, de maio de 2003 a maio de 2004, foram realizadas amostragens na vegetação espontânea presente entre as plantas de citros e nas entrelinhas, em 30 pontos sorteados. Em cada ponto recolheu-se uma unidade de amostra na linha e outra na entrelinha, num total de 60 unidades por ocasião. Cada unidade consistiu de todas as folhas com minas das plantas presentes num círculo de $0,28 \mathrm{~m}^{2}$, delimitado por um aro de pvc com $60 \mathrm{~cm}$ de diâmetro, adaptação feita do método do quadrilátero, proposto por Southwood (1978).

As plantas atacadas por minadores foram retiradas com auxílio de uma pá de jardineiro. Quandonão era possível retirar toda a planta, ramos foram recolhidos com uma tesoura de poda. As plantase/ou ramos foram colocados, individualmente, em sacos plásticos identificados, os quais foram transportados até o laboratório em caixa de isopor contendo termogel.

No laboratório as folhas foram examinadas em estereomicroscópio, registrando-se o número de larvas e/ou pupas de dípteros minadores. As folhas foram acondicionadas em placas de Petri de $9 \mathrm{~cm}$ de diâmetro e $1,5 \mathrm{~cm}$ de altura e/ ou em caixas gerbox de $11,2 \mathrm{~cm}$ de diâmetro e $3,4 \mathrm{~cm}$ de altura, mantidas em câmara climatizada (fotofase de 12 horas, $25^{\circ} \mathrm{C} \pm 1^{\circ}$ C) a té a emergência dos adultos de minadores e/ou de parasitóides. Para manter a turgidez das folhas, seus pecíolos foram envolvidos por um chumaço de algodão que era umedecido diariamente.

Os parasitóides e os dípteros emergidos foram conservados individualmente em recipientes do tipo "eppendorf" contendoálcool etílico hidratado a 92, $8^{\circ}$ o qual foi diluído a 70\% de água.

A identificação das famílias dos parasitóides foi efetuada com o auxílio da chave dicotômica deGAULD; 
Hanson (1995) e a identificação em nível genérico e/ ou específico foi feita por especialistas, assim como os exemplares de dípteros. À exceção dos exemplares retidos pelos especialistas, todos os exemplares de dípteros minadores e de parasitóides obtidos foram depositados na coleção de referência do Departamento de Fitossanidade, Faculdade de Agronomia, da UFRGS.

A comunidade de parasitóides associada aos dípteros minadores foi descrita pela riqueza de espé- cies, número absoluto e freqüência relativa de cada espécie.

\section{RESULTADOS E DISCUSSÃO}

Foram obtidas 15 espécies de himenópteros parasitóides (134 indivíduos), distribuídas em três famílias, associadas à fase larval ou pupal de onze espécies de dípteros minadores (Tabela 1).

Tabela 1 - Parasitóides emergidos e dípteros minadores amostrados em vegetação espontânea em pomar de tangoreiro Murcott, no município de Montenegro, RS (maio de 2003 a maio de 2004).

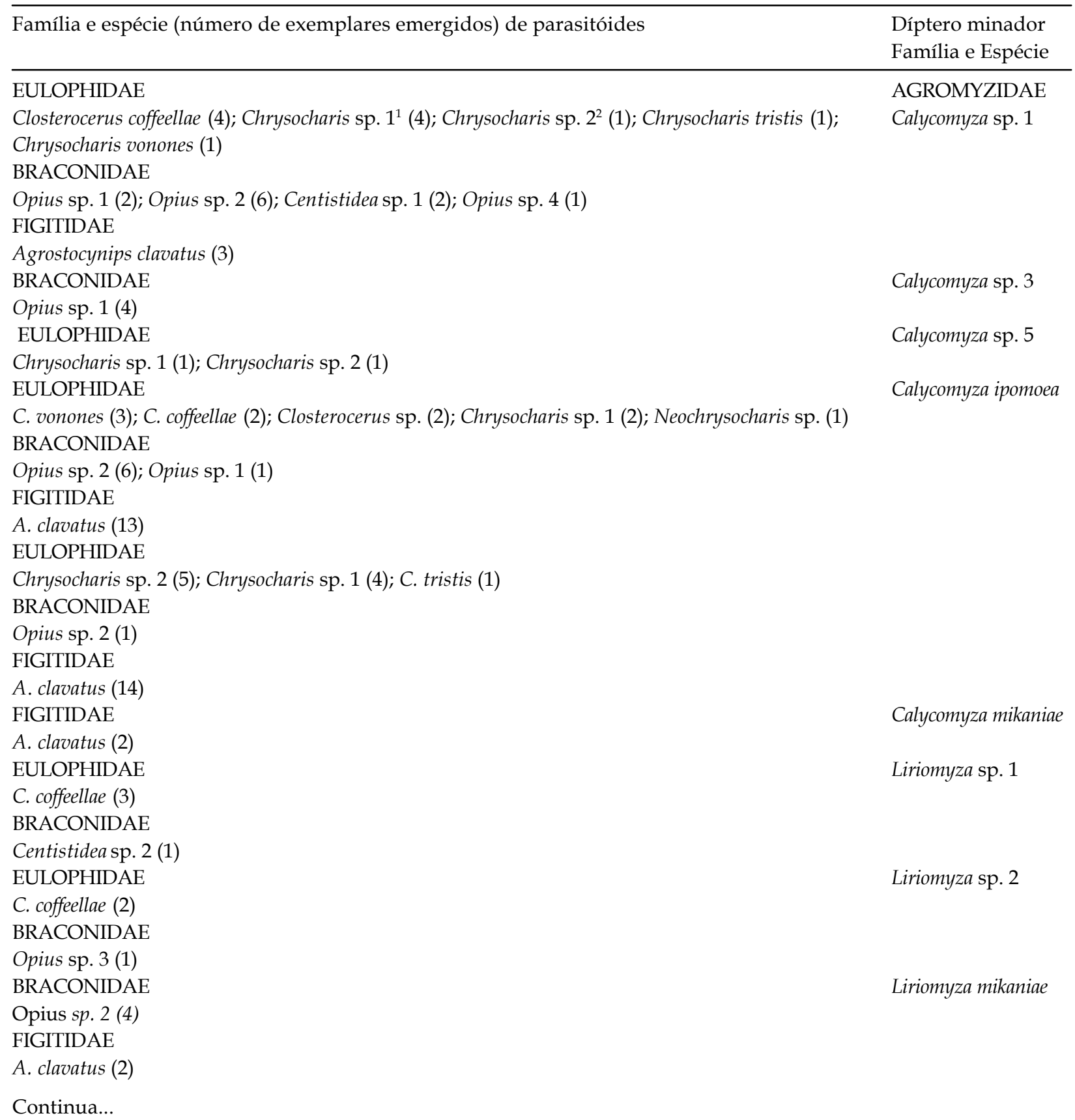


Tabela 1 - Continuação

\section{EULOPHIDAE}

C. vonones (3); Chrysocharis sp. 1 (1); Chrysocharis sp. 2 (1); C. tristis (1)

\section{BRACONIDAE}

Opius sp. 1 (16); Centistidea sp. 1 (2); Opius sp. 5 (1)

\section{FIGITIDAE}

A. clavatus (6)

EULOPHIDAE

Chrysocharis sp. 1 (2)

Agromyza sp.

1,2São, provavelmente, novas espécies.

Onúmero deespécies de parasitóides foi crescente ao longo das amostragens (Fig. 1A) e, até a $23^{\text {a }}$ amostragem, ainda estavam sendo registradas novas espécies, o que sugere que este número poderia ainda ser maior na área de estudo com a continuidade das amostragens. Onúmero de famílias de parasitóides se estabilizou a partir da $3^{\text {a }}$ amostragem (Fig. 1B), o que indica que o número registrado representa o presente na área.

Analisando a Tabela 1, verifica-se que as espécies de Eulophidae, Braconidae e Figitidae obtidas podem ser parasitóides tanto de Calycomyza spp. (Diptera: Agromyzidae) quanto de Liriomyza spp. Eulophidae apresentou o maior número de espécies - sete, distribuídas pelos gêneros Chrysocharis Förster (Entedoninae), Closterocerus Westwood (Entedoninae) eNeochrysocharis Kurdjumov (Entedoninae), com 46 indivíduos. Foram amostradas sete espécies de Braconidae (48 exemplares) pertencentes aos gênerosOpius Wesmael(Opiinae) e Centistidea Haliday (Miracinae).

Dentre os parasitóides emergidos de dípteros minadores, Agrostocynips clavatus Díaz (Figitidae) apresentou maior frequiência relativa (Fig. 2).

Associadas às espécies de Calycomyza foram encontradas 12 espécies de himenópteros parasitóides, distribuídas em três famílias (Tabela 1). A. clavatus foi o parasitóide com a maior freqüência relativa $(36,3 \%)$, seguida deOpius sp. $2(14,8 \%)$,
Chrysocharis sp. 1 (12,5\%), Chrysocharis sp. 2 (8,0\%) e Opius sp. $1(8,0 \%)$, Closterocerus coffeellae Ihering $(6,8 \%)$, Chrysocharis vonones Walker $(4,5 \%)$, Centistidea sp. 1, Chrysocharis tristis Hansson e Closterocerus sp. (2,3\% cada) e,Neochrysocharis sp.e Opius sp. 4 (1,1\% cada).

Da mesma forma, associadas àLiriomyza spp. também foram registradas 12 espécies de himenópteros parasitóides, distribuídas em três famílias (Tabela 1). Opius sp. $1(36,4 \%)$ foi a mais freqüente, seguida por $A$. clavatus $(18,2 \%)$, C. coffeellae $(11,4 \%)$, Opius sp. 2 (9\%), C. vonones (6,8\%), Centistidea sp. 1 (4,5\%). As espécies Chrysocharis sp. 1 e Chrysocharis sp. 2, Centistidea sp. 2, C. tristis, Opius sp. 3 eOpius sp.5apresentarama mesma frequiência relativa $(2,3 \%$ cada).

Associados à Agromyza sp. foram obtidos somente dois indivíduos de Chrysocharis sp. 1.

HANSSON; GAULD (1995) relataram que Eulophidae é a terceira família de Chalcidoidea (depois de Aphelinidae e Encyrtidae) em importância para o controle biológico, apresentando as espécies mais utilizadas no controle de insetos minadores de Coleoptera, Diptera, Hymenoptera e Lepidoptera, destacando-se as dos gêneros Chrysocharis, Closterocerus e Neochrysocharis (Clausen, 1940; HANSSON, 1995a, b;SCHAUFF etal.,1997). Na sua maioria são endoparasitóides solitários de larvas ou pupas (HANSSON, 1985; HANSSON, 1994).
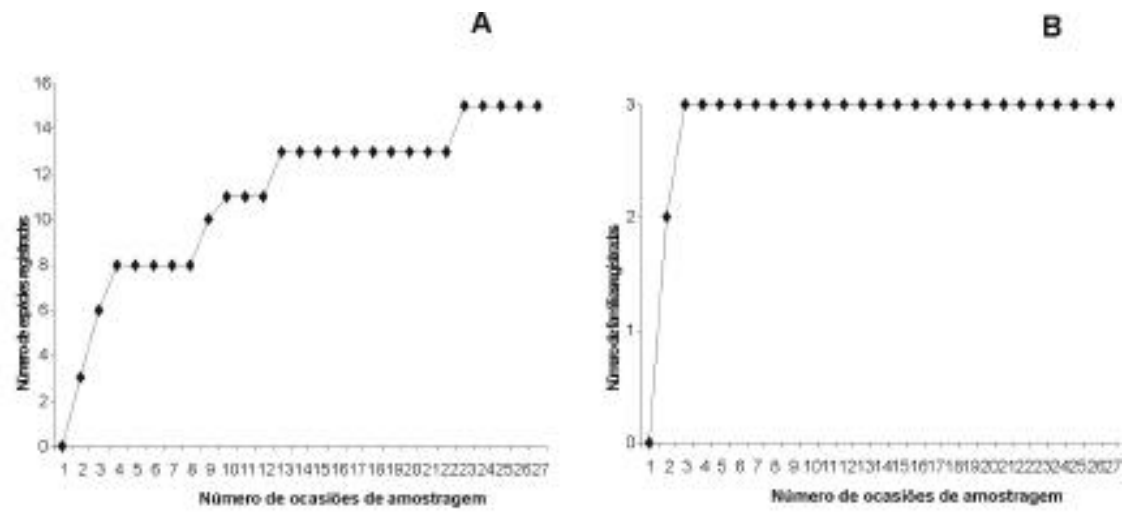

Fig. 1 - Número cumulativo de (A) espécies e (B) de famílias de himenópteros parasitóides, obtidas em sucessivas amostragens em vegetação espontânea, em pomar de tangoreiro Murcott, no Município de Montenegro, RS (maio de 2003 a maio de 2004). 


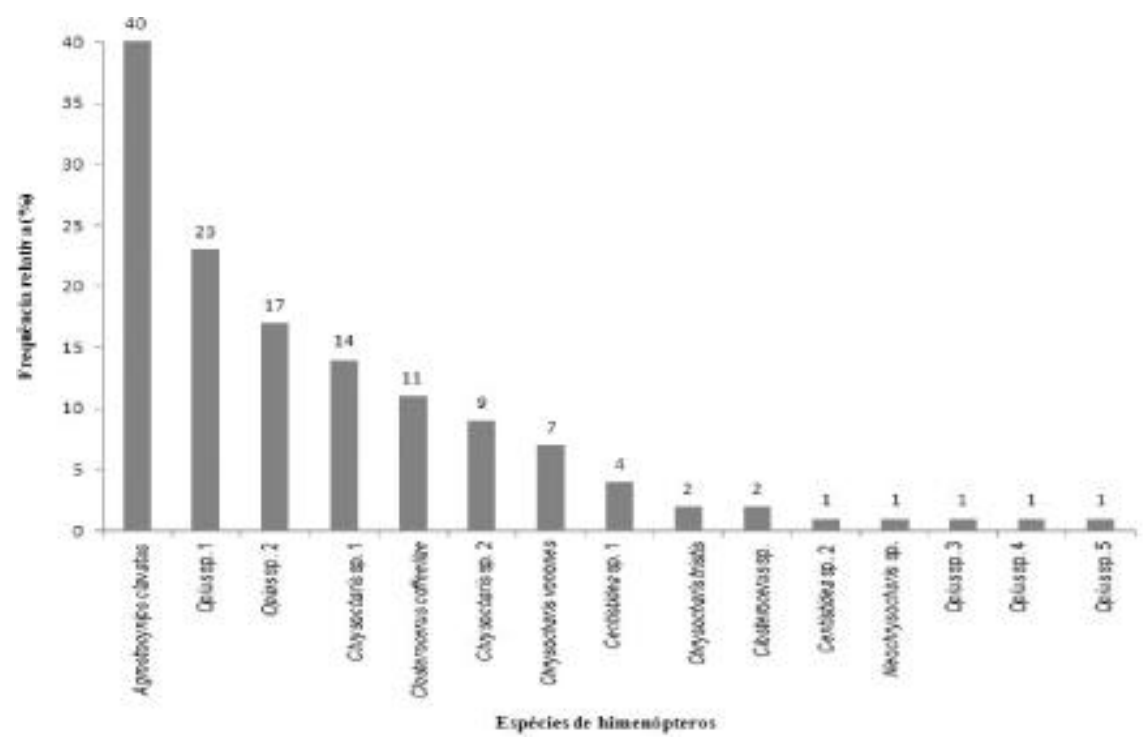

Fig. 2 - Freqüência relativa das espécies de himenópteros parasitóides emergidas de dípteros minadores e número de indivíduos (expressos no topo das colunas) amostrados em vegetação espontânea, em pomar de tangoreiro Murcott, no Município de Montenegro, RS (maio de 2003 a maio de 2004).

De acordo com Hansson (1995a), as espécies de Neochrysocharis apresentaram vasta gama de hospedeiros, inclusive dípteros agromizídeosque vivemem plantas espontâneas e pastagens.

Segundo Clausen (1940), C. coffeellae tem hábito gregárioe as fêmeas podem ovipositar mais deumovo por hospedeiro. No presente estudo registraram-se três adultos de C. coffeellae por indivíduo deLiriomyza sp. 1, dois/indivíduo de Liriomyza sp. 2, um/indivíduo de Calycomyza sp. 1 e 2,3 por indivíduo de Calycomyza ipomoea (Frost) (Diptera: Agromyzidae).

Foram amostradas sete espécies de Braconidae (48 exemplares) pertencentes aos gênerosOpiusWesmael, 1835 (Opiinae) e Centistidea Haliday (Miracinae) (Tabela 1).

Opiinae é uma das maiores subfamílias de Braconidae, com mais de 1.300 espécies descritas, a maioria incluída em Opius (WHARTon, 1997). Espécies deste gênero são parasitóides larvais-pupais solitários (SCHUSTER; WHARTON, 1993).

Segundo Wharton (1997), espécies de Opius são amplamente distribuídas em todos os continentes; nas Américas ocorrem do Canadá a Argentina e, na região Neártica, são utilizadas no controle biológico de moscas-das-frutas e de agromizídeos minadores. Opius spp. têm grande diversidade de hospedeiros, destacando-se as espécies de Liriomyza de várias partes do mundo (SCHUSTER et al., 1991;SCHUSTER; WHARTON,1993).

Em Miracinae foram registradas duas espécies de Centistidea. De acordo com Müsевеск (1937), poucos estudos têm sido realizados com membros desta subfamília devido à escassez de espécimes amostrados. Penteado-Dias (1999) referiu-se que espécies de Centistidea são endoparasitóides de larvas, porém, emergem quando os hospedeiros empupam. Apenas uma espécie de Figitidae (Eucoilinae, Eucolini) foi obtida: A. clavatus com 40 indivíduos emergidos (Tabela 1). Os membros desta família são endoparasitóides cenobiontes de larvas de grande número de dípteros agromizídeos e de moscas-dasfrutas (GuimARÃes et al., 2003).

Cabe salientar que, apesar de C. coffeellae ter ocorrido em dípteros minadores, verificou-se neste caso que esta espécie não foi a mais abundante.

Parasitóides pertencentes a alguns destes gêneros constatados no presente estudo já haviam sido referidos com espécies associadas a Liriomyza spp. por Schusteretal. (1991), Valladares;SALvo (2001) ePereiRA et al. (2002).

Dentre as espécies de parasitóides amostradas associadas a dípteros minadores, há algumas incluídas nos gêneros: Chrysocharis (SCHAuff et al., 1998; Urbaneja et al., 1998); Closterocerus (BRowning; PeÑa, 1995; PeÑa et al., 1996; Perales-Gutierrez et al., 1996; SCHAUfF et al., 1998; CANCINO et al., 2001; LegaSpi et al., 1999; LegASPI et al., 2001) e Neochrysocharis (Legaspi et al., 1999) com relatos de parasitismo sobre P. citrella.

No pomar orgânico estudado, a vegetação espontânea representou um hábitat importante para uma grande diversidade de dípteros minadores e associados a estes, e várias espécies de parasitóides foram registradas. Estes resultados evidenciam quea manutenção da vegetação espontânea nos sistemas agrícolas tem um papel importante como abrigo para inimigos naturais. $\mathrm{O}$ manejo adequado desta vegetação pode favorecer tanto a conservação de espécies benéficas, como o controle biológico natural daquelas consideradas pragas. 


\section{AGRADECIMENTOS}

Ao biólogo Luís Laux, por ter concedido a área de realização do estudo; ao Dr. Thomas Michael Lewinsohn (UNICAMP), pelo encaminhamento dos dípteros para identificação; à Dra. Graciela Valladares (Centro de Investigaciónes Entomológicas de Córdoba, Argentina), pela identificação dos dípteros minadores; ao Dr. Valmir Antonio Costa (Instituto Biológico), pela confirmação e identificação de algumas espécies de parasitóides; ao Dr. Christer Hansson (Department of Zoology, University of Lund, Suécia), pela identificação de Chrysocharis spp., Neochrysocharis sp. e Closterocerus spp., assim como pelo fornecimento de bibliografia; à Dra .Angélica Maria Penteado-Dias (UFSCar), pela confirmação das espécies de Opius; ao Dr. Jorge Anderson Guimarães (EMBRAPA-CNPAT), pela identificação de Agrostocynips clavatus; às bolsistas de iniciação científica Rita de Cássia Antochevis e Ester Foelkel (UFRGS), pelo auxílio na realização dos trabalhos de campo e de laboratório e, ao $\mathrm{CNPq}$, pelas bolsas concedidas ao primeiro e terceiro autores.

\section{REFERÊNCIAS}

BYERS, J.A. Leaf-mining insects 2002. Disponível em: <http://www. wcrl.ars.usda.gov/cec/insects/ leafmine. htm>. Acesso em: 27 dez. 2004.

BROWNING, H.W.; PEÑA, J.E. Biological control of the citrus leafminer by its native parasitoids and predators. Citrus Industry, v.76, p.46-48, 1995.

CANCINO, E.R.; BERNAL, C.M.; BLANCO, M.C.; CRESPO, J.R.M.; PEÑA, J.E. Hymenopteros parasitoides de Phyllocnistis citrella Stainton (Lepidoptera: Gracillariidae) en Tamaulipas y Norte de Veracruz, México, con una clave para las especies. Folia Entomologica Mexicana, v.40, n.1, p.83-89, 2001

CARLETTI, E. Insectos de Argentina y el mundo. Disponível em: <http://www.axxon.com.ar/mus/info/ art-DondeHallarInsectos.htm>. Acesso em: 27 dez. 2004.

CLAUSEN, C. P. Entomophagous insects. New York: McGraw-Hill, 1940. 688p.

CURE, J.R.; CANTOR, F. Atividade predadora e parasítica de Diglyphus begini (Ashm.) (Hymenoptera: Eulophidae) sobre Liriomyza huidobrensis (Blanch.) (Diptera: Agromyzidae) em cultivos de Gysophila paniculata L. Neotropical Entomology, v.32, n.1, p.85-89, 2003.

GALLO, D.; NAKANO, O.; SILVEIRA NETO, S.; CARVALHO, R.P.L.; BAPTISTA, G.C.; BERTI FILHO, E.; PARRA, J.R.P.; Zucchi, R.A.; Alves, S.B.; VendRAMIM, J.D.; MARChinI, L.C.; Lopes, J.R.S.; ОмоTo, C. Entomologia agrícola. Piracicaba: FEALQ, 2002. 920p.
GAULD, I.D.; HANSON, P.E. The evolution, classification and identification of the Hymenoptera. In: Hanson, P.E.; GAULD, I.D. The Hymenoptera of Costa Rica. Oxford: Oxford University Press, 1995. p.138156.

GLIESMANN, S.R.; GARCIA, E.R.; AMADOR, A.M. The ecological basis for the application of traditional agricultural technology in the management of tropical agroecosystems. Agroecosystems, v.7, p.173-185, 1981. Gravena, S. Lagarta minadora dos citros no Brasil. Laranja, v.17, n.1, p.286-288, 1996.

GUIMARÃES, J.A.; GALLARDO, F.E.; DIAZ, N.B.; ZUCCHI, R.A. Eucoilinae species (Hymenoptera: Cynipoidea: Figitidae) parasitoids of fruit-infesting dipterous larvae in Brazil: identify, geographical, distribuition and host associations. Zootaxa, v.278, p.123, 2003.

HANSSON, C. Taxonomy and biology of the Palearctic species of Chrysocharis Förster, 1856 (Hymenoptera: Eulophidae). Entomologica Scandinavica Supplement, v.26, p.1-130, 1985.

HANSSON, C. Re-evaluation of the genus Closterocerus Westwood (Hymenoptera: Eulophidae) with a revision of the Nearctic species. Entomologica Scandinavica, v.25, n.1, p.1-26, 1994.

HANSSON, C. Revision of the Nearctic species of Neochrysocharis Kurdjumov (Hymenoptera: Eulophidae). Entomologica Scandinavica, v.26, n.1, p.27-46, 1995a.

HANSSON, C. Revised key to the Nearctic species of Chrysocharis Förster (Hymenoptera: Eulophidae), including three new species. Journal Hymenoptera Research, v.4, p.80-98, 1995b.

HANSON, P.E.; GAULD, I.D. The Hymenoptera of Costa Rica. Oxford: University Press, 1995. 893p.

HESPENHEIDE, H.A. Bionomics of leaf-mining insects. Annual Review of Entomology, v.36, p.535-560, 1991.

LEGASPI, J.C.; FRENCH, J.V.; SCHAUFF, M.E.; WOOLLEY, J.B. The citrus leafminer Phyllocnistis citrella (Lepidoptera: Gracillariidae) in South Texas: incidence and parasitism. Florida Entomologist, v.82, p.305-316, 1999.

LEGASPI, J.C.; FRENCH, J.V.; ZUÑIGA, A.G.; LEGASPI JÚNIOR, B.C. Population dynamics of the citrus leafminer, Phyllocnistis citrella (Lepidoptera: Gracillariidae), and its natural enemies in Texas and México. Biological Control, v.21, p.84-90, 2001.

LEÓN, A.; PINO, M. DE LOS A.; GONZÁLEZ, C.; DEL POZO, E. Evaluación comparativa de densidades de fitófagos y enemigos naturales en policultivo tomatemaíz. Cultivos Tropicales, v.21, n.1, p.53-60, 2000. 
MUESEBECK, C.F.W. A new West Indian species of Mirax Haliday parasitic on the coffee leaf-miner (Hymenoptera: Braconidae). Proceedings of the Entomological Society of Washington, v.39, p.139-141, 1937.

PARRA, J.R.P.; BOTELHO, P.S.M.; CORREAA-FERREIRA, B.S.; BENTO, J.M.S. Controle biológico: terminologia. In: PARRA, J.R.P.; BOTELHO, P.S.M.; CORRÊAFERREIRA, B.S.; BENTO, J.M.S. (Ed.). Controle biológico no Brasil: parasitóides e predadores. São Paulo: Manole, 2002. p.1-16.

PATEL, K.J.; SCHUSTER, D.J.; SMERAGE, G.H. Density dependent parasitism and host-killing of Liriomyza trifolli (Diptera: Agromyzidae) by Diglyphus intermediatus (Hymenoptera: Eulophidae). Florida Entomologist, v.86, n.1, p.8-14, 2003.

PEÑA, J.E.; DUNCAN, R.; BROWNING, H.W. Seasonal abudance of Phyllocnistis citrella (Lepidoptera: Gracillariidae) and its parasitoids in South Florida citrus. Environmental Entomology, v.25, n.3, p.698-702, 1996.

PENTEADO-DIAS, A.M.; GRAVENA, S.; PAIVA, P.E.B.; PINTO, R.A. Parasitóides de Phyllocnistis citrella (Stainton) (Lepidoptera: Gracillariidae: Phyllocnistinae) no Estado de São Paulo. Laranja, v.18, n.1, p.79-84, 1997.

PERALES-GUTIÉRREZ, M.A.; ARREDONDO-BERNAL, H.C.; GARZA-GONZÁLEZ, E.; AGUIRRE- URIBE, L.A. Native parasitoids of citrus leafminer Phyllocnistis citrella Stainton in Colima, México. Southwestern Entomologist Scientific, v.21, n.3, p.349-350, 1996.

PEREIRA, D.I. DA P.; SOUZA, J.C. DE; SANTACECÍLIA, L.V.C.; REIS, P.R.; SOUZA, M. de A. Parasitismo de larvas da mosca-minadora Liriomyza huidobrensis Blanchard (Diptera: Agromyzidae) pelo parasitóide Opius sp. (Hymenoptera: Braconidae) na cultura da batata com faixas de feijoeiro intercaladas. Ciência e Agrotecnologia, v.26, n.5, p.955-963, 2002.

SCHAUFF, M.E.; LASALLE, J.; COOTE, L.D. EULOPHIDAE. IN: GIBSON, G.A.P.; HUBER, J.T.; WOOLLEY, J.B. (Ed.). Annotated keys to the genera of nearctic Chalcidoidea (Hymenoptera). Ottawa: National Research Council Research Press, 1997. p.327-429.
SCHAUFF, M.E.; LASALLE, J.; WIJESEKARA, G.A. The genera of the Chalcid parasitoids (Hymenoptera: Chalcidoidea) of citrus leafminer Phyllocnistis citrella Stainton (Lepidoptera: Gracillariidae). Journal of Natural History, v.32, p.1001-1056, 1998.

SCHUSTER, D.J.; WHARTON, R.A. Hymenopterous parasitoids of leaf-mining Liriomyza spp. (Diptera: Agromyzidae) on tomato in Florida. Environmental Entomology, v.22, n.5, p.1188-1191, 1993.

SOUTHWOOD, T.R.E. Ecological methods: with particular reference to the study of insect populations. London: Chapman and Hall, 1978. 524p.

URBANEJA, A.; JACAS, J.; VERDÚ, M.J.; GARRIDO, A. Dinámica e impacto de los parasitoides autóctonos de Phyllocnistis citrella Stainton, en comunidad valenciana. Investigación Agraria: Producción y Protección Vegetales, v.13, n.3, p.409-423, 1998.

VALLADARES, G.; SALVO, A. Community dynamics of leafminers (Diptera: Agromyzidae) and their parasitoids (Hymenoptera) in a natural habitat from Central Argentina. Acta Oecologica, v.22, p.301-309, 2001.

VAN EMDEN, H.F. The role of uncultivated land in the biology of crop pests and beneficial insects. Scientia Horticulturae, v.17, p.121-136, 1964.

VENETTE, R.C.; HUTCHISON, W.D.; BURKNESS, E.C.; O'ROURKE, P.K. El minador de la hoja de la alfafa: actualización de la investigación. Disponível em: <http://www.ipmword.umn.edu/cancelado/ spchapters/venettesp.htm>. Acesso em: 10. out. 2003.

VERDÚ, M.J. Chalcidoidea (Hymenoptera), parásitos del minador de los cítricos Phyllocnistis citrella (S) (Lep.; Gracillariidae) en España. Levante Agrícola, v.336, p.227230, 1996.

WHARTON, R.A. Subfamily Opiinae. In: WHARTON, R.A.; MARSH, P.M.; SHARKEY, M.J. (Ed.) Manual of the New World genera of the Family Braconidae (Hymenoptera). Lawrence: The International Society of Hymenopterists, 1997. p.379-395.

Recebido em $1 / 8 / 08$

Aceito em 12/8/08 